\title{
Germanica
}

\section{Violences sur les scènes allemandes}

Gewalt auf deutschen Bühnen

\section{Eliane Beaufils}

\section{(2) OpenEdition}

Journals

Édition électronique

URL : http://journals.openedition.org/germanica/326

DOI : 10.4000/germanica.326

ISSN : 2107-0784

Éditeur

Université de Lille

\section{Édition imprimée}

Date de publication : 1 décembre 2006

Pagination : 103-115

ISBN : 2-913857-18-3

ISSN : 0984-2632

Référence électronique

Eliane Beaufils, "Violences sur les scènes allemandes », Germanica [En ligne], 39 | 2006, mis en ligne le 19 février 2010, consulté le 06 octobre 2020. URL : http://journals.openedition.org/germanica/326 ;

DOI : https://doi.org/10.4000/germanica.326

Ce document a été généré automatiquement le 6 octobre 2020.

(c) Tous droits réservés 


\title{
Violences sur les scènes allemandes
}

\author{
Gewalt auf deutschen Bühnen
}

\section{Eliane Beaufils}

1 Depuis le début des années 1990, les scènes sanglantes, brutales ou pornographiques se multiplient dans les théâtres allemands. À en juger par les rangées de fauteuils qui se vident parfois, elles peuvent incontestablement faire violence au spectateur. Mais elles ne semblent guère pouvoir être mises sur le compte d'une nouvelle esthétique de l'effroi dans le prolongement d'Heiner Müller : en effet, les auteurs n'entendent pas symboliser les abus de pouvoir ni les crimes de la civilisation occidentale dans son ensemble, à de rares exceptions près comme Elfriede Jelinek, qui ne saurait cependant être comptée parmi les jeunes écrivains de la nouvelle génération ${ }^{1}$. Certains dramaturges s'inscrivent évidemment dans la lignée de leurs augustes prédécesseurs de l'entre-deux-guerres et des années 1960-1970, Piscator, Brecht, Fassbinder, Peter Weiss ou Franz Xaver Kroetz. Ils allient comme eux la violence dénoncée à la violence de la dénonciation: nombreuses sont les œuvres notamment qui condamnent après la réunification les actes criminels d'extrême-droite, telles Rabenland d'Hans-Jörg Schertenleib ou Wessis in Weimar de R. Hochhuth, ainsi que les mentalités arriérées subsistant en Allemagne, telle Die schöne Fremde de Klaus Pohl ${ }^{2}$. Les pièces se font l'écho de l'impuissance ressentie par une majorité de la population, laquelle se sent tenue d'endiguer cette violence sans savoir comment procéder. Mais, sous couvert de l'impuissance, elles aboutissent souvent à une apologie esthétique de la violence qui n'est pas dépourvue d'ambiguité, d'autant que bon nombre d'entre elles ne sont guère travaillées sur un plan formel. Les seules à promouvoir sans ambages la distance critique et la réflexion sont finalement les pièces pour jeunes, qui adoptent des parti pris ostensiblement brechtiens ${ }^{3}$ - quasi-absence de scènes violentes susceptibles de provoquer des émotions, songs, techniques de récapitulation ou de « commentaire »- , ou misent sur les interventions du jeune public et les échanges ${ }^{4}$.

2 La majeure partie des pièces violentes ne peut malgré tout être située dans le prolongement du théâtre politique, populaire ou expressionniste. Elles sont en vérité difficiles à aborder : non seulement aucun message clair n'est associé à la violence, mais les effets ne peuvent en aucun cas être réduits à la terreur ou à la pitié. Ces œuvres 
peuvent nous plonger dans la perplexité en ne nous présentant plus la violence comme "l'Autre de l'homme» et de la société ${ }^{5}$, y compris par le moyen de la provocation. Celle-ci n'est-elle pas encore une manière de s'élever contre la violence parées des traits de la légitimité, du pouvoir ou de la conformité? Ces nouvelles pièces contreviennent donc à toute conception "schillérienne » du théâtre et ne paraissent pas non plus être de simples œuvres de transgression. On peut se demander si elles ne vont pas au-delà de déconstructions supplémentaires pour expérimenter un autre homme autant qu'un « autre théâtre » (Antonin Artaud'). Elles s'essayent de fait à des écritures extrêmement diverses.

3 Le mode expérimentatoire caractéristique du théâtre contemporain empêche plus que jamais de s'en tenir au "fond" et à "la forme ", puisque les lumières et les gestes deviennent tous des signes autonomes, et tout mot devient son, geste ou poème. La violence, pierre angulaire de notre appréhension de l'humain, semble non seulement livrée à l'activité du récepteur, mais ne saurait rentrer dans le cadre de taxinomies sémiologiques hâtives. De plus, l'approche de la violence peut différer d'une œuvreà l'autre chez un même auteur. Aussi nous ne présenterons ici que trois dramaturges, plus ou moins représentatifs des trois principales tendances, sans prétendre à aucune exhaustivité dans la présentation de cette violence théâtrale ouverte. Nous analyserons cependant la démarche de Werner Schwab de manière plus précise, parce que le jeune trublion du début des années 1990 a révolutionné l'approche de la violence, si bien que nombre de pièces ultérieures sont marquées du sceau "schwabien »... quand bien même les auteurs prennent radicalement leurs distances avec les modèles de tous ordres.

\section{Werner Schwab, écrivain de l'abject}

4 Werner Schwab fait partie des rares écrivains à avoir, comme ses aînés Elfriede Jelinek et Peter Turrini, défrayé la chronique. Il a dans le même temps peut-être été le dernier à faire scandale. Il semble effectivement qu'il soit difficile d'aller plus loin dans la représentation d'actes outranciers que le jeune Autrichien ${ }^{7}$ À lui plus qu'à tout autre, on a fait le reproche de la gratuité, de l'esthétisme et d'une joie sadique sans autres fins qu'eux-mêmes ${ }^{8}$. Nous pouvons quant à nous nous déprendre des formules contradictoires de l'auteur ou des expressions assassines des journaux de l'époque. Nous tenterons ainsi d'évaluer la pertinence ou la gratuité des effets et des moyens employés par rapport à la conception du théâtre schwabien et de celle de l'homme qui lui est liée.

$5 \quad$ Les pièces de Werner Schwab se caractérisent au premier chef par une activité scénique qui déjoue systématiquement nos pires craintes. Dans ses deux premiers et principaux succès, Enfin mort, enfin plus de souffle et Extermination du peuple ou Mon foie n'a pas de $s^{s e n} s^{9}$, il est fait mention de meurtres horribles : les clients du bar très mal fréquenté $\mathrm{d}$ ' Enfin mort, enfin plus de souffle s'en prennent tous au beau jeune couple qu'ils dévorent tandis que la digne Madame Grollfeuer finit par « exterminer " tous les locataires qui partagent l'immeuble avec elle. Quant aux dernières pièces de l'auteur, elles ne tendent plus simplement à un climax de l'horreur mais s'en nourrissent sans discontinuer, mettant en acte les sodomies et autres tortures simplement évoquées dans les pièces antérieures. Au triomphe de la force et de la traîtrise, il n'est opposé aucune alternative. Les personnages les plus faibles sont souvent ridicules et bêtes, comme 
Mariedl dans Les Présidentes ou Helmut Brennwert dans Escalade ordinaire, ce sont des "vers estropiés $»^{10}$ qui alimentent la haine dont ils sont l'objet, sans qu'il n'y ait au demeurant de raison objective à leur humiliation et mise à l'écart radicale. Les personnages les plus ambigus esquissent des itinéraires tragiques, tels Brennwert ou la Mariedl d'AnticlimaX, mais ne sauraient paraître dignes de pitié tant leur sentimentalisme ou leur participation aux méfaits les intègrent malgré tout dans le système de la subjectivité déchaînée et sadique. On pourrait penser que le caractère manifestement arbitraire de tous les actes finit par leur enlever leur dimension agressive: mais il s'agit tellement d'actes contre nature, i.e. contre-culture ${ }^{11}$, qu'ils gardent un effet "neuromimétique» sur le spectateur ${ }^{12}$. De plus, le corps des personnages reste, semble-t-il, toujours pourvu d'une signification: il s'agit d'une signification que nous attachons au corps en soi et qui découle de notre première appréhension du moi ${ }^{13}$. Cette signification, dont nous ne pouvons pour d'évidentes raisons perceptives et émotives liées à l'ensemble de notre expérience, nous défaire, fait l'objet de multiples débats dans le cadre des études théâtrales, en vertu de la dimension de plus en plus performative du théâtre ${ }^{14}$. Mais comme Schwab feint encore de respecter le principe de figuration, par lequel un personnage figure une personne dotée d'une identité, la violence apparaît toujours comme atteinte à l'intégrité et à la volonté d'autrui. Lorsque la victime fait montre de douleur surtout, comme cela arrive chez Brennwert et chez Mariedl, la pièce institue le spectateur en témoin.

Heureusement, les procédés comiques et grotesques du langage constituent une soupape, aussi radicale que l'est la violence corporelle et mentale. De fait, la langue de Werner Schwab est dotée de particularités tellement remarquables qu'elle a d'office été qualifiée de "schwabisch» - en référence également au dialecte "grossier » du pays souabe et des pièces populaires critiques. Or cette langue est de plus en plus difficile à saisir au fur et à mesure des pièces, et la complexité formelle en général accompagne le recours à des actes de plus en plus odieux. Maints critiques ont ainsi simplement réduits les dernières pièces à une surenchère des moyens éprouvés auparavant. La dynamique de l'écriture est néanmoins plus subtile.

7 D'une part, Werner Schwab s'appuie sur des procédés comiques on ne peut plus traditionnels: redondances, répétitions, exagérations, anthropomorphisation des objets ou objectivation des êtres humains. Ils rappellent les inversions carnavalesques et le théâtre populaire, usant de la troisième personne au lieu de la première. Ces procédés reposent cependant pour beaucoup sur des innovations importantes, des néologismes surabondants, surchargés de suffixes et de préfixes, redondants ou oxymoriques, des groupes nominaux et des subordonnées foisonnantes qui dérèglent complètement la syntaxe. Le dramaturge autrichien est incontestablement influencé, comme Elfriede Jelinek, par l'avant-garde formaliste viennoise, les travaux d'Oscar Wiener et de Konrad Bayer. Il s'appuie, autre tradition autrichienne, sur la combinaison du sensuel et du morbide, des aliments et des excréments, la mise en avant des protubérances et des ouvertures du corps, ainsi complètement éclaté et non harmonieusement clos sur lui-même. Or ces éléments propres au grotesque (Bakhtine) sont menés à une telle extrémité, la langue est tellement défigurée, logorrhéique et comme noyée dans les redondances improbables qu'elle se dissout, devient magmatique et oppose une résistance. Le langage devient endocannibale, en sorte qu'on se concentre malgré tout sur les personnages, lesquels s'apparentent finalement à des mélodies, ponctuées par le retour obsédant de mots-clés, de parties physiologiques et /ou d'actions. Il s'agit là encore d'une démarche voisine de celle de 
maints dramaturges autrichiens, Karl Kraus, Elias Canetti ou encore Elfriede Jelinek, qui font apparaître les personnages comme des marionnettes et des masques, si ce n'est que le caractère mélodique et charnel des mots investit les personnages schwabiens d'une corporalité et d'une présence plus grande.

Ce langage dote aussi les idéologèmes d'une portée unique. Toutes les compositions à base de justice, d'honnêteté, d'État, de capital, de justice ou d'éducation tournent à vide comme une ritournelle nihiliste destructrice: les personnages se renvoient réciproquement leurs reproches de "chiennerie », « injustice » ou "criminalité » si bien qu'en dénonçant le fonctionnement arbitraire de l'idéologie, qui n'est jamais que le masque de la domination, ils lui enlèvent au fur et à mesure du texte toute substance. Il ne s'agit pas d'une table rase cependant, car dans cet univers où le sol se dérobe constamment sous les pieds du spectateur, subsiste l'invocation des mots-clés obsédants et destructeurs, subsistent les références au bon, au religieux ou au sentiment qui trouvent encore des traces d'accomplissement dans les personnages christiques ou antéchristiques principaux. Si le spectateur est plongé dans le magma de «l'indéfinitude ", il ne saurait pour autant tout relativiser, c'est là l'insigne cruauté des pièces. Sous le couvert du nihilisme grotesque et absurde, elles confrontent à des mots vitaux, à une violence pulsionnelle et enferment le spectateur dans leur fonctionnement. Le dégoût des aliments et de soi qui se fait jour dans la langue et les actes - Mariedl court par exemple se fracasser la tête contre les murs -, le dégoût du père symbolique et plus encore de la mère, qui sont les principales marques de l'abject, le « contaminent ».Au-delà de la circularité oppressante de la langue et de la scène, le spectateur est pris dans les filets de l'abject. Mais le rire qui transparaît dans les "éclats de la langue ${ }^{15}$ et de la scène lui permet de ne pas y plonger, il doit « seulement » affronter l'étrangement inquiétant (« l'Unheimliche »), à la fois proche (heimlich) et si lointain ${ }^{16}$. La violence schwabienne permet donc de faire accéder le spectateur de manière performative à sa négativité telle que la définit Julia Kristeva, cette qualité insigne de l'écrivain qui confronte ses pulsions sémiotiques au thétique, au symbolique existant, et accède à une symbolisation supérieure grâce à laquelle il intègre les dynamiques sémiotiques « révolutionnaires » et essentielles. La corporalité paradoxale de la théâtralité si travaillée de Schwab est donc affectée d'une vraie cruauté dans l'esprit d'Antonin Artaud. Le déchirement des forces, de l'impensé fait chair et langue amène le spectateur à la "vie supérieure de la scène ", dotée d'une dimension à la fois expérimentatoire et quasi-métaphysique, cosmique.

Très nombreux sont les dramaturges qui se réclament de Werner Schwab : on peut citer Franzobel, Bernhard Studlar ou Kathrin Röggla. Si la plupart d'entre eux se tournent vers un théâtre critique, dont la dimension n'est pas étrangère à Schwab eu égard aux idéologèmes tragiques et religieux auxquels il reste attaché, la fascination du modèle a souvent conduit à des formulations inspirées de Schwab, chez Thomas Jonigk par exemple, ou à des actions scéniques morbides et nihilistes. Or ces moyens ne sont pas des recettes et la question reste posée de savoir si on peut (se) confronter à l'abject sans faire preuve d'une intense négativité comme Werner Schwab. Il semble bien plutôt que les parti pris de Robert Woelf $\left.\right|^{17}$ soient caractéristiques d'une autre tendance en voie de développement, plus actuelle que celle du dramaturge autrichien mort il y a déjà plus de dix ans. 


\section{Le théâtre du mal de Robert Woelfl}

10 Entre Robert Woelfl et Werner Schwab, il y a d'abord une considérable rupture de style qui ne manque pas de porter à conséquence. Le langage de Woelfl est non seulement très clair mais très simple. L'intrigue obéit à des principes dramatiques et non postdramatiques, si bien que les personnages et l'action semblent reproduire la réalité de manière assez mimétique. On a l'impression a priori que toutes les voies de distanciation usuelles ou moins usuelles font défaut, et que les activités scéniques ne sauraient avoir de potentiel critique. Au contraire, si stylisation il y a, elle paraît résider dans ce subtile crescendo univoque vers le pire: au fur et à mesure des pièces de Woelfl, les personnages dévoilent leurs perversités, tant et si bien qu'une horreur permet de préparer la suivante. Dans Einmal satt, einmal tot, einmal gesellig (qui rappelle le titre de Schwab Endlich tod, endlich keine Luft mehr ${ }^{18}$ ), les personnages sont tous dénués de joie, de sensibilité et de spontanéité, mais remplis d'envies destructrices qu'ils rêvent tout haut de satisfaire. Ils finissent par donner corps à des cauchemars vivants ou par assister aux crimes des autres en les oubliant aussitôt : les deux jeunes en mal d'action obligent un couple de gens aisés et ennuyés à ingérer le cadavre d'un chien en le dépeçant d'abord avec leurs dents, le vieux pêcheur les regarde sans intervenir, et quand il découvre le cadavre de la jeune fille tuée par les mêmes jeunes, il le viole alors que le meurtrier s'immole comme il l'avait prévu dès le début. La méchanceté et la brutalité semblent consubstantielles à l'être humain. Dans les premières pièces comme Einmal satt, elles épargnent néanmoins certains personnages: ainsi la jeune Sabine, terrifiée par le châtiment imposé au couple bourgeois, et obligée de fuir pour ne pas se sentir mal - ce qui lui vaut d'être assassinée -, incarne une réaction normale. Mais le jeune amoureux idéaliste de Wahrheit ${ }^{19}$, trompé volontairement par la belle et son meilleur ami, finit par leur ressembler. Il succombe finalement au désabusement mortifère de son ami, incarné par la drogue et une anesthésie complète envers les sentiments. Si l'anéantissement physique n'est qu'esquissé dans cette pièce, il accompagne dans les autres l'anéantissement de l'identité morale et affective.

D'aucuns ont voulu voir poindre une trace d'ironie précisément dans le manque d'ironie affiché de l'auteur, au travers de l'exagération voilée, de la surprise que ménagent sans cesse les actions. Certes, l'incongruité peut avoir un effet comique, mais cet effet est infiniment plus grand et plus recherché chez un dramaturge comme Wilfried Happel, dont les pièces sont de véritables comédies sanglantes, jouant sur tous les effets dramatiques possibles. L'invraisemblable de R. Woelfl n'obéit pas à la logique de l'escalade absurde s'appuyant sur des motifs connus. La perversité est bien plutôt synonyme de créativité : ainsi le vieux couple dans Kommunikation der Schweine ${ }^{20}$ feint de vouloir être torturé par un tiers, qu'il engage, alors qu'il agit de la sorte pour mieux retourner la situation, tant et si bien que l'homme engagé est dévoré par deux cannibales aguerris. Le meilleur ami de l'amoureux dans Wahrheit crée aussi la réalité à son image. La perversité est même le signe de la liberté, voire de la libération dans Dem Herz die Arbeit, den Händen die Liebe ${ }^{21}$, car tous les personnages se jouent les uns des autres et réduisent les forts en esclavage. Cette dimension créatrice s'étend aux victimes telles le couple bourgeois cynique et blasé, qui renaît à une vie propre après avoir déchiqueté le cadavre canin et ne saurait plus passer pour caricatural.

12 Vis-à-vis de ces pièces, publiées par une maison d'édition de renom et représentées dans les plus grands théâtres d'Allemagne, la réaction des journalistes est révélatrice : 
la plupart d'entre eux s'entendent à reconnaître une dimension critique à la violence chez Woelfl, mais n'en introduisent pas moins des scrupules en mentionnant le caractère problématique de l'univocité des pièces. Sans nul doute on assiste à un malaise dans le positionnement, imputable d'abord au préjugé favorable dont jouissent les pièces prétendument sérieuses, perçues d'emblée comme des prolongements du théâtre politique antérieur. Voilà qui rejoint l'avis de R. Willemsen sur le cinéma. Celuici raille les journalistes qui s'en prennent aux films d'horreur mais qui omettent de remettre en cause les images violentes des films grand public ou des films d'auteur, car elles seraient d'emblée affectées d'un label critique ${ }^{22}$.

L'absence de prise de position véritable des critiques ne manifesterait-elle pas finalement un certain goût pour les antivaleurs, pour un théatre "interdit » ou " antischillérien »? Il se peut que ce genre de pièces illustre l'opinion de K.-H. Bohrer, selon laquelle une bonne partie des œuvres allemandes rattraperaient aujourd'hui un des «manques» de la modernité allemande, celui d'une littérature transgressive, opposée au canon de valeurs dominant, notamment à la recherche d'une identité allemande accompagnée de culpabilité (protestante, catholique et viennoise, et surtout politique $)^{23}$.

Optant pour des parti pris formels quasiment inverses de ceux de Woelfl, ostensiblement postdramatiques, un autre groupe de pièces est habité par le souci de se déprendre de toutes les traditions littéraires antérieures. Il fait œuvre de "déconstruction» pour que, dans l'impossibilité radicale de se référer à une expérience et appréhension personnelle du monde, le spectateur se saisisse de tous les signes et redécouvre une autre réalité. Est-ce que l'appréciation de la violence est tout entière livrée au récepteur en ce cas ou dévoile-t-elle encore une cruauté « artaudienne » de la vie? Pour mieux aborder ce groupe de pièces, quantitativement de très loin dominant, nous nous appuierons notamment sur la pièce d'Albert Ostermaier, The Making of. B-Movie, qui présente l'avantage de réfléchir le succès même des pièces « trash » en Allemagne.

\section{Le théâtre de la violence déréalisée}

Albert Ostermaier fait d'ores et déjà partie des jeunes dramaturges de renom, dont chaque nouvelle pièce constitue un événement depuis les deux premières ${ }^{24}$.The Making of. constitue cependant la première pièce où l'auteur quitte le registre de la poésie dramatique, désormais réservé à ses recueils de poèmes, en campant des personnages résolument cyniques, insaisissables et souvent violents, caractéristiques des pièces suivantes. Comme cette pièce représente par ailleurs une satire des milieux culturels, elle appréhende de manière singulièrement systématique ce qu'il faut bien appeler « la raison cynique " contemporaine ${ }^{25}$, et fait clairement comprendre les causes ou motivations de la violence, ce qui n'est pas le cas des pièces postérieures, moins systématiques et plus allusives.

The Making of. traite de deux amis acteurs, Silber et Brom : Silber, un acteur allemand renommé, vient chercher son ami Brom, un poète méconnu réfugié en Afrique, qui doit endosser pour lui le rôle du parfait trublion : il doit s'afficher comme un mercenaire, poète et dramaturge, qu'aurait découvert Silber et qui étale de manière provocante sa vulgarité et sa luxure. Silber escompte ainsi faire scandale et fortune à travers sa créature en diffusant ses propres œuvres. Mais la créature a raison de son maître, 
l'évince, détourne son œuvre et le poignarde une fois arrivée au faîte de la gloire. " Le coup [des deux charlatans] réussit donc, [...] car les intellectuels allemands aiment des types comme [Brom], des terroristes, qui feignent de réconcilier l'art et l'authenticitée ${ }^{6}$ ».

17 De fait, la dimension satirique se manifeste d'emblée par le recours à des caricatures : tout en étant des sortes d'actualisations populaires des premières pièces de Brecht, des personnages de Baal notamment, elles témoignent de l'impossibilité de l'authenticité. Que ce soit le mécène, l'amie de la mécène qui devient la maîtresse de Brom, son amant masculin Silber, le critique d'art interloqué puis intéressé par l'affaire, l'actrice Hannah, tous, hormis le maître d'hôtel Johannes, se caractérisent par leur cynisme : il ne s'agit pas cependant d'un cynisme machiavélien, sceau du pouvoir et du secret, ni d'un nihilisme révolutionnaire auquel on a parfois voulu réduire Baal, mais de la raison cynique. Celle-ci naît de ne pouvoir adhérer à un système de références, à un discours de légitimation, a fortiori à une raison absolue. Chacun est livré à la multitude contradictoire des références, est «l'agent de plusieurs discours $»^{27}$ : Hannah par exemple se récrit contre la barbarie de Brom qui veut lui faire dépecer un cadavre de cheval sur scène, car l'art lui semble obéir à des impératifs plus ou moins schillériens, mais elle revient en lui lançant sa chevelure rasée en pleine figure pour jouer de nouveau, séduite malgré elle par l'appât du gain et du succès scandaleux. On use des discours en vertu des circonstances et des intérêts. Les formes les plus fréquentes sont le cynisme mercantile, qui, pour Sloterdijk, s'offre de nos jours de lui-même et à tous, mais aussi le cynisme vis-à-vis de la violence, et en particulier de la guerre: le mercenaire Brom permet, sous couvert d'authenticité, de laisser libre cours à la fascination de la violence et à celle de la guerre faite dans des pays lointains pour asseoir notre bonne conscience.

Or le cynisme produit une forme d'atomisation du moi, il devient difficile de saisir une personnalité au-delà de l'instant et des apparences. Comme tout repose sur le simulacre et la manipulation originelle de Brom et Silber, le spectateur ne sait bientôt plus à quoi s'en tenir, ni qui manipule qui. Il est d'autant plus insécurisé que le message critique se retourne contre lui-même: tout en dénonçant les travers des contemporains, The Making Of. n'en vérifie pas moins le succès des pièces «trash » en Allemagne. De plus, Ostermaier l'intitule «B-Movie» en multipliant les associations faciles, film de série « B », bas de gamme, pseudo-brechtien, où lui-même rappelle la figure provoquante de Brecht et de maints autres jeunes auteurs adulés. Il fait un véritable pied de nez à son commanditaire, le vénérable théâtre d'État de Munich (Staats-schauspielhaus), et à l'establishment culturel munichois.

Mais ce sont les choix esthétiques qui achèvent de donner à la satire une tonalité particulière : l'auteur demande de multiplier les écrans de télévision et grands écrans, si bien que tout est en même temps théâtre dans le film, film dans le théâtre, émission de télévision dans le théâtre, réfléchie indéfiniment. La multiplication des perspectives contribue à brouiller les pistes, à tout relativiser et à tout déréaliser. Non seulement elle va à l'encontre du principe de présence et de vie qui est au fondement du théâtre mimétique, mais le spectateur doit jongler avec des rôles démagogiques et démultipliés, une somme d'abstractions où rien n'importe plus vraiment. Ainsi il est confronté à un flux d'informations sans véritable tension dramatique. La déréalisation n'est donc pas simplement une critique constructive parce qu'elle reflèterait les conditions de vision et d'écoute de la société médiatique ${ }^{28}$. 
20 Ces processus ont bien entendu des implications radicales sur la perception de la violence. Celle-ci devient elle-même part du jeu, simulacre, elle consacre la "dissolution de la valeur ${ }^{29}$. Le spectateur a une liberté d'appréciation presque totale, qui culmine à la fin où trois conclusions sanglantes lui sont proposées. Il assiste à une tentative de meurtre de Silber par Brom à la fin de la représentation barbare des amazones, se voit ensuite contraint d'assigner un autre statut au légionnaire qui est le double de Silber et s'en prend à Brom, réfugié dans un bar en Afrique, au point de le mettre à mort; enfin, lors de la grande cérémonie qui fête le triomphe théâtral de Brom et Silber, le faux dramaturge poignarde Silber au vu et au su de tous en faisant passer son acte pour une mise en scène. Or l'impossibilité de croire à un réel enjeu final, qui plus est vital, de croire donc à l'acte dramatique par excellence, risque de plonger le spectateur dans l'indifférence, ou du moins, dans la « nonchalance ${ }^{30}$.

21 Le théâtre obéit alors au principe de la télévision, où «tout peut toujours advenir en permanence ${ }^{31}$. La mort, et dans une moindre mesure la violence, n'est plus que le "dernier principe d'adversité $»^{32}$ dans un monde dissous, où rien n'est plus tout à fait positivé. Autrement dit, un reste de sens quand le sens fuit infiniment. Cela apparaît mieux dans cette pièce que dans d'autres d'Albert Ostermaier, où le meurtre final est davantage le fruit du hasard et de l'indétermination des personnages, comme dans Auf Sand. Car dans The Making of. (dans Katakomben aussi) se dessine encore une passion, une passion de s'affirmer, de vivre face à autrui et face à soi. Dans la plupart des autres pièces, la conscience n'est plus que de l'ordre de la résignation. La manière de s'y livrer aux contingences sans vouloir se livrer au hasard fait naître précisément la violence.

22 Évidemment, ce théâtre déréalisé plus qu'onirique est problématique. Au cynisme ambiant on oppose une sorte de cynisme esthétique, coupant court à l'émotion, au désir, à la participation, à la responsabilité. Et l'indifférence de ce théâtre souvent profondément "non-érotique $"^{33}$ menace de gagner le spectateur tout comme le conformisme menace de gagner la violence. La spécificité performative du théâtre s'efface, notamment quand il s'agit de faire passer cette passion de la survie qui se déclare avec la violence.

On peut observer en ce moment une tendance à revenir à des esthétiques beaucoup plus dépouillées, plus à même de restaurer le temps du théâtre, l'épreuve d'une mortalité en suspens et le sentiment de la vie malgré l'atomisation et le caractère factice des actions. C'est le cas par exemple de la pièce Der Bus de Lukas Bärfuss ${ }^{34}$ : tout est inscrit dans un contexte cynique, et même méchant, comme chez R. Woelfl, car les passagers d'un bus de tourisme s'en prennent tous à la jeune pélerine montée à bord par erreur. Mais d'une part, l'enlisement de l'action au fond d'une forêt perdue l'affecte d'une dimension irréelle, d'autre part l'itinéraire de la pélerine se détache de plus en plus du bus, elle fait des expériences humaines profondes qui transcendent le contexte de départ et le font passer au second plan. De telles œuvres restent beaucoup moins « négatives » cependant que les œuvres de Werner Schwab et elles ne restaurent pas de cruauté envers le spectateur. On pourrait d'ailleurs craindre que le scepticisme global qui menace aujourd'hui l'acte de création théâtral ne l'élude pour longtemps, si la cruauté ne relevait précisément d'une impulsion créatrice personnelle, et qu'elle peut advenir à tout moment comme elle le fait chez Werner Schwab ou Elfriede Jelinek. 


\section{NOTES}

1. Il faut noter toutefois qu'Elfriede Jelinek a seulement développé l'écriture dramatique dans les années 1980 et que chacune de ses pièces peut être conçue comme un véritable laboratoire, au point que l'écriture théâtrale est désormais son mode d'expression privilégié, sanctionné entre autres par l'attribution à deux reprises du célèbre prix dramatique de Mülheim en 2002 et 2004.

2. Hans-Jörg Schertenleib, Rabenland, (1993) ; Rolf Hochhuth, Wessis in Weimar (1993); Klaus Pohl, Die schöne Fremde (1991)

3. On pourra se reporter par exemple à la pièce du Grips-Theater, Hallo Nazi, rédigée en 2001 par le collectif anonyme MONOBLOCK, anonyme par peur d'éventuelles représailles !

4. Voilà le principe essentiel du "théâtre de participation " (Mitmachtheater) du théâtre Rote Grütze de Berlin. On se réfère en particulier à leur pièce Gewalt im Spiel, qui date cependant de 1988.

5. Gilbert Kirschner, Figures de la violence et de la modernité. Essais sur la philosophie d'Eric Weil, Lille : Presses Universitaires de Lille, 1992

6. A. Artaud, Le Théâtre et son double, Paris : Gallimard, 1938, 1964

7. On rappelle que W. Schwab, né en 1957, a connu une gloire foudroyante en 1991 et jusqu'à sa mort le $1^{\text {er }}$ janvier 1994. Durant cette période, l'auteur n'a cessé d'écrire, sur commande ou de son propre chef, après d'intenses années d'isolement. Durant les années 1980 en effet, il s'était retiré en Styrie près de sa ville natale Graz, et avait écrit en prose ou conçu des œuvres plastiques à base de matières putrescibles qui font écho à la langue de ses pièces.

8. Cf. On renvoie à cette phrase souvent citée: "le point de départ du théâtre, il est étonnamment indifférent »./ «[Es] ist erstaunlich gleichgültig, wovon ein Theater ausgeht », in W. Schwab, « Vorwort », Fäkaliendramen, Graz : Droschl, 1991, p. 10

9. Titres originaux: Endlich tot, endlich keine Luft mehr et Volksvernichtung oder meine Leber ist sinnlos, tous deux parus in Werner Schwab, Fäkaliendramen, Graz: Droschl, 1991. Les traductions sont de Mike Sens et Michael Bugdahn, pour les éditions de L'Arche, Paris, respectivement parues en 1997 et en 2000.

10. Thomas Trenkler, «Die Krüppelwürmer: der Defekt in den Dramen von Werner Schwab », in Roland Duhamel, Clemens Ruthner (Hrsg), 2. Republik- 1000 Jahre "Ostarrichi », Bonn: Dümmler, 1996, p. 165-174.

11. Les deux se rejoignent car la plupart des incestes, sodomies, masturbations et meurtres publics sont condamnés par toutes les cultures comme le fait remarquer Hans-Peter Dürr dans son ouvrage anthropologique et historique de synthèse, Der Mythos vom Zivilisationsprozess. 3: Obszönität und Gewalt, Frankfurt a.M.: Suhrkamp, 1993.

12. L'expression est de Stanton B.Garner, Bodied Spaces. Phenomenology and Performance in Contemporary Drama. Ithaca and London, 1994.

13. Cf. Lacan: "L'acteur ne prête pas seulement ses membres, sa présence au personnage théâtral comme s'il était une marionnette, il réalise le rôle à l'aide de son inconscient réel, i.e. de la relation de ses membres à sa propre histoire ». In J. Lacan, Hamlet, « Le désir de la mère » : Le Séminaire livre VI, in Ornicar 25, 1982, p. 18.

14. Cf. par exemple: « [ Man muss] nachhaltige Zweifel daran [haben], der Körper könne jenseits von Signifikanz auf der Bühne erscheinen ». A. Opel s'oppose à l'idée des gestes « libres de sens » (« sinnfrei ») telle que la soumet H. T. Lehmann : même s'ils ne s'intègrent pas dans une logique narrative, ils déclenchent toujours un processus de décryptage sémiotique. Cf. Anna Opel, Sprachkörper: Zur Relation von Sprache und Körper in der zeitgenössischen Dramatik, Bielefeld: Aisthesis Verlag, 2002, p. 33-34 et H.T. Lehmann, Postdramatisches Theater, Frankfurt: Verlag der Autoren, 1999, p. 366. 
15. Julia Kristeva, La Révolution du langage poétique, Paris : Seuil, 1974.

16. «Das Unheimliche » a fait l'objet d'un premier texte de Freud (1919) et de maintes études sur les processus de création. Cf. Notamment Bernard Chauvier, «Le paradoxe intimiste et la création", in B. Chauvier, Symbolisation et processus de création: sens de l'intime et travail de l'universel dans l'art, Paris : Dunod, 1998, p. 127-157.

17. Robert Woelfl est né en 1965 à Villach en Carynthie (Autriche). Il se met à écrire après avoir suivi des études en arts plastiques, et a notamment reçu le prix Lenz de la ville de Jena en 2000. Il vit à Vienne et ses pièces sont toutes publiées chez Fischer.

18. Cf. R. Woelfl, Einmal satt, einmal tot, einmal gesellig, Frankfurt : Fischer, 1999 et W. Schwab, cité note 9 .

19. R. Woelfl, Wahrheit, Frankfurt : Fischer, 2002.

20. R. Woelfl, Kommunikation der Schweine, Frankfurt : Fischer, 2000.

21. R. Woelfl, Dem Herz die Arbeit, den Händen die Liebe, Frankfurt: Fischer, 1999.

22. Roger Willemsen, « Gewalt als Unterhaltung », in Merkur, Heft 2, 1985, p. 91-105.

23. K-H. Bohrer, «Die permanente Theodizee », in Imaginationen des Bösen, München: Hanser, 2004, p. 33-62.

24. L'auteur, né en 1967 à Munich, fête à vrai dire son premier succès dès 1995 avec ses albums de poésie, Nicht in Venedig et Herz Vers Sagen, respectivement parus en 1991 et en 1995, pour lesquels il obtient le prix du Pen Liechtenstein. Du jour au lendemain, il devient par ailleurs un dramaturge renommé grâce à sa pièce dédiée à Ernst Toller, Zwischen zwei Feuern. Tollertopographie, représentée en juin 1995 à Munich, puis grâce à Tatar Titus. Il a été auteur invité par le théâtre de Munich en 1998-1999 pour écrire The Making of. et reçoit depuis chaque année un prix ou un contrat d'écriture en résidence.

25. En référence à l'expression du célèbre ouvrage de Peter Sloterdijk, Kritik der zynischen Vernunft, Frankfurt : Suhrkamp, 1983.

26. «Der Coup gelingt [...], weil die deutschen Intellektuellen Typen wie [Brom] lieben, Chaoten, die Authentizität in die Kunst zu bringen vorgeben », C. Bernd Sucher, "Ab morgen bist du eine Kampfmaschine! W. Minks inszeniert die Uraufführung von A. Ostermaiers ,The Making Of. BMovie' im Münchner Cuvilliétheater », in Süddeutsche Zeitung, 01.06.1999.

27. Cf. « multiple Agentenschaft», in P. Sloterdijk, cité note 24, p. 223.

28. C'est notamment l'opinion de H. T. Lehmann, cité note 14.

29. Baudrillard, La Transparence du mal. Essai sur les phénomènes extrêmes, Paris : Galilée, 1990.

30. H.T. Lehmann, ibid.

31. Baudrillard, ibid.

32. Baudrillard, ibid.

33. « Nichterotisches Theater », Peter Kümmel, in Die Zeit, 12/2001.

34. Cette pièce a été primée au festival de Mülheim 2005.

\section{RÉSUMÉS}

À l'origine de cet article, il y a le constat d'une apparition de plus en plus fréquente de la violence sur les scènes allemandes et autrichiennes. S'agit-il d'un épiphénomène sans portée particulière, témoin d'une impasse idéologique et du besoin de faire concurrence aux arts audiovisuels ? Ou est-ce là le reflet d'une mutation plus profonde de notre vision de l'humain, intégrant une 
cruauté jusqu'alors reléguée au rang de tabou? On assiste peut-être à une application spontanée des théories d'Antonin Artaud, lequel entendait dans les années 1930 promouvoir un théâtre de la cruauté, mais remettait également en question le vieil antagonisme entre culture et violence. Aussi nous attachons nous aux oeuvres qui ne semblent plus du tout relayer l'optique antagoniste traditionnelle en condamnant la violence qu'elles portent sur la scène : au contraire, les pièces de Werner Schwab, de Robert Woelfl et d'Albert Ostermaier s'abstiennent de tout jugement unilatéral sur la violence. Ces trois démarches n'épuisent pas la variété des écritures en jeu, argument s'il en faut de leur subjectivité et du caractère si vivant du théâtre en Allemagne. Mais elles permettent de saisir la portée de trois choix esthétiques qui, par delà la violence, renvoient à des visions différrentes du théâtre et de la culture : une vision éminemment cruelle dont la férocité n'a d'égale que la singularité, et des visions cyniques dont le cynisme ne se réduit pas à des postures. Jamais néanmoins le spectateur ne peut échapper à la performativité de la violence « en jeu », à l'espace des possibles qu'elle ouvre comme un gouffre au sein de la vie du théâtre, moins que jamais rassurante et close sur la belle apparence.

Die immer häufiger auf den deutschen und österreichischen Bühnen auftretenden Gewaltakte stellen den Ausgangspunkt der gegenwärtigen Analyse dar. Es fragt sich, ob es sich hierbei nur um ein Epiphänomen ohne sonderliche Tragweite handelt, das Zeugnis einer ideologischen Sackgasse ist, und um das Bedürfnis, mit den audiovisuellen Medien zu konkurrieren, oder ob es vielmehr um eine tiefergreifende Vision des Menschlichen geht, das eine bisher ins Tabu verlegte Grausamkeit widerspiegelt. Vielleicht kann man in diesem Phänomen aber auch eine spontane Anwendung der Theorien von Antonin Artaud sehen, der in den dreißiger Jahren ein Theater der Grausamkeit fördern, gleichzeitig aber auch den alten Antagonismus zwischen Kultur und Gewalt in Frage stellen wollte. Die im vorliegenden Artikel besprochenen Werke scheinen die Vision des traditionellen Antagonismus keineswegs ablösen und die auf die Bühne getragene Gewalt verdammen zu wollen. Die Stücke von Werner Schwab, Robert Woelfl und Albert Ostermaier verzichten im Gegenteil auf jegliches einseitiges Urteil über die Gewalt. Ihre Art, mit der Gewalt umzugehen, sind nur drei Modelle, die keineswegs die mannigfaltigen Schreibtechniken, Subjektivitäten und Charaktere des höchst lebendigen Theaterbetriebs in Deutschland erschöpfen. Sie erlauben es indes, die Tragweite dieser drei ästhetischen Modelle zu erfassen, die jenseits von aller Gewalt auf verschiedenartige Visionen des Theaters und der Kultur verweisen, auf eine höchst grausame sowie auf zynische Visionen, die sich nicht auf reine Posituren begrenzen lassen. Der Zuschauer kann jedoch nie der Performativität der « ins Spiel » geratenen Gewalt entkommen: Er vermag sich nicht dem Raum des Möglichen zu entziehen, das sich ihm wie ein Abgrund inmitten des nie mehr tröstenden und schönen Scheins gewidmeten Theaterlebens auftut.

\section{INDEX}

Mots-clés : théâtre, violence

oeuvrecitee Fäkaliendramen, Antiklimax, einmal satt, einmal tot, einmal gesellig, Wahrheit, Kommunikation der Schweine, Dem Herz die Arbeit, Den Händen die Liebe, The making of

\section{AUTEURS}

ELIANE BEAUFILS

Hambourg 